\title{
Some bifurcation routes to chaos of thermocapillary convection in two- dimensional liquid layers of finite extent
}

Article in Physics of Fluids · May 2016

DOI: $10.1063 / 1.4948400$

CITATIONS

0

3 authors, including:

K. Li

Chinese Academy of Sciences

34 PUBLICATIONS 136 CITATIONS

SEE PROFILE
READS

27

Bo Xun

Chinese Academy of Sciences

9 PUBLICATIONS 30 CITATIONS

SEE PROFILE

Some of the authors of this publication are also working on these related projects:

Convection and its stability during the formation of polymer hollow fiber membranes by spinning process View project 


\title{
AIP $\mid$ Physics of Fluids
}

\section{Some bifurcation routes to chaos of thermocapillary convection in two- dimensional liquid layers of finite extent}

\author{
K. Li, B. Xun, and W. R. Hu
}

Citation: Physics of Fluids 28, 054106 (2016); doi: 10.1063/1.4948400

View online: http://dx.doi.org/10.1063/1.4948400

View Table of Contents: http://scitation.aip.org/content/aip/journal/pof2/28/5?ver=pdfcov

Published by the AIP Publishing

\section{Articles you may be interested in}

The influence of the interfacial heat release on nonlinear convective oscillations in two-layer systems

Phys. Fluids 25, 072106 (2013); 10.1063/1.4813607

Two-dimensional numerical analysis of electroconvection in a dielectric liquid subjected to strong unipolar injection

Phys. Fluids 24, 037102 (2012); 10.1063/1.3685721

Three-dimensional oscillatory long-wave Marangoni convection in a binary liquid layer with the Soret effect: Bifurcation analysis

Phys. Fluids 19, 072105 (2007); 10.1063/1.2749305

Influence of thermocapillary effect and interfacial heat release on convective oscillations in a twolayer system

Phys. Fluids 16, 1127 (2004); 10.1063/1.1652654

Oscillatory and chaotic thermocapillary convection in a half-zone liquid bridge

Phys. Fluids 15, 408 (2003); 10.1063/1.1531993

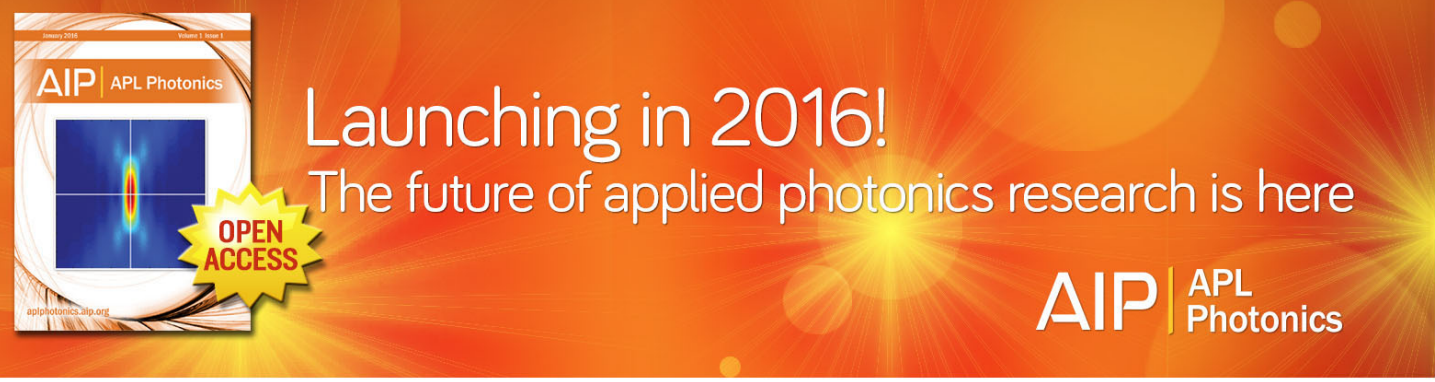




\title{
Some bifurcation routes to chaos of thermocapillary convection in two-dimensional liquid layers of finite extent
}

\author{
K. Li, ${ }^{1,2, a)}$ B. Xun, ${ }^{1}$ and W. R. Hu ${ }^{1}$ \\ ${ }^{1}$ Key Laboratory of Microgravity, Chinese Academy of Sciences, Beijing 100190, China \\ and National Microgravity Laboratory, Institute of Mechanics, Chinese Academy of Sciences, \\ Beijing 100190, China \\ ${ }^{2}$ University of Chinese Academy of Sciences, Beijing 100190, China
}

(Received 13 July 2015; accepted 14 April 2016; published online 9 May 2016)

\begin{abstract}
As a part of the preliminary studies for the future space experiment (Zona-K) in the Russian module of the International Space Station, some bifurcation routes to chaos of thermocapillary convection in two-dimensional liquid layers filled with $10 \mathrm{cSt}$ silicone oil have been numerically studied in this paper. As the laterally applied temperature difference is raised, variations in the spatial structure and temporal evolution of the thermocapillary convection and a complex sequence of transitions are observed. The results show that the finite extent of the liquid layer significantly influences the tempo-spatial evolution of the thermocapillary convection. Moreover, the bifurcation route of the thermocapillary convection changes very sensitively by the aspect ratio of the liquid layer. With the increasing Reynolds number (applied temperature difference), the steady thermocapillary convection experiences two consecutive transitions from periodic oscillatory state to quasi-periodic oscillatory state with frequency-locking before emergence of chaotic convection in a liquid layer of aspect ratio 14.25 , and the thermocapillary convection undergoes period-doubling cascades leading to chaotic convection in a liquid layer of aspect ratio 13.0. Published by AIP Publishing. [http://dx.doi.org/10.1063/1.4948400]
\end{abstract}

\section{INTRODUCTION}

Turbulent phenomenon is inherent in nature. ${ }^{1,2}$ The bifurcation routes to chaos for a buoyant natural convection have attracted extensive studies. ${ }^{3-11}$ Typical bifurcation routes that might announce chaos are identified, such as frequency-locking, ${ }^{4,7}$ period-doubling route, ${ }^{4,8}$ the RuelleTakens-Newhouse route, ${ }^{4,9,10}$ and the intermittent route, ${ }^{4,11}$ depending on the controlling parameters of the dissipative dynamical systems such as the Rayleigh number, the Prandtl number, and the geometric aspects. Different from the buoyant natural convection, thermocapillary convection is the principal natural convection in microgravity or in shallow liquid layers in the terrestrial conditions in which the capillary effect is dominant. As a relatively new dissipative dynamical system, thermocapillary convection has become one of the fundamental subjects in microgravity fluid physics and space fluid/heat management. ${ }^{12-14}$ The liquid layer is usually adopted as one of the typical models for the investigation on the thermocapillary convection, which is driven by the surface tension gradient induced by the temperature gradient along the free surface. Smith and Davis ${ }^{15,16}$ studied the stability of an infinite liquid layer driven by imposed temperature gradient parallel to the free surface in case of zero gravity. They found stationary longitudinal rolls and unsteady hydrothermal wave for the case with flat and non-deformable free surface and surface wave for the case with deformable free surface. The mechanism of the hydrothermal wave is further explained by Smith. ${ }^{17}$ Thereafter, the hydrothermal wave instability has received considerable attentions, and many experimental and numerical studies associate with the hydrothermal wave have been performed. Thermocapillary convection and its stability have been experimentally studied in shallow liquid layers in the

\footnotetext{
${ }^{a)}$ Electronic mail: likai@imech.ac.cn
} 
terrestrial conditions by many researchers. ${ }^{18-22}$ However, the experiments on the thermocapillary convection in the terrestrial conditions are complicated by the coupling with buoyancy effect. For instance, the results shown by Riley and Neitzel reveal that the characteristics of the hydrothermal wave (angle of propagation, frequency of oscillation) are different for liquid layers with different thickness, ${ }^{18}$ which may be due to the effect of gravity as studied by Chan and Chen ${ }^{23}$ On the other hand, there are few space experiments on the problem. Kamotani et al. studied the steady and oscillatory thermocapillary convection in cylindrical containers experimentally on board the Space Shuttle. ${ }^{24,25}$ The liquid layer used is relatively deep with aspect ratio around 1. Schwabe et al. investigated thermocapillary convection and its stability in a large and shallow annular gap heated from the outer wall. ${ }^{26,27}$ Under high supercritical condition, the thermocapillary convection will bifurcate further until evolve to chaos convection. Few pioneer experiments on secondary instability of hydrothermal waves in horizontal pools under high super-criticality were reported. ${ }^{28-30}$ Mukolobwiez et al. studied the nonlinear dynamics of hydrothermal waves in a long and thin annular channel heated from the side and showed a supercritical Eckhaus instability. ${ }^{28}$ Garnier et al. investigated the nonlinear dynamics of waves and modulated waves in long and narrow 1D channels (annular or bounded) and presented the observations in the framework of complex Ginzburg-Landau equations. ${ }^{29,30}$ There are also many numerical investigations associated with the thermocapillary convection and its instability. Carpenter and Homsy performed a linear stability analysis of the thermocapillary convection in a rectangular liquid pool. ${ }^{31} \mathrm{Xu}$ and Zebib numerically simulated the two-dimensional (2D) and three-dimensional (3D) thermocapillary convection in rectangular liquid layers under zero gravity condition. ${ }^{32} \mathrm{Ma}$ and Bothe studied the thermocapillary convection in liquid layer with dynamically deformable interface by direct numerical simulation based on the volume of fluid method. ${ }^{33}$ Sáenz et al. studied the linear and nonlinear stability of hydrothermal waves in shallow planar liquid layers driven by thermocapillarity by means of two-phase direct numerical simulations taking into account the dynamical interfacial deformation. ${ }^{34}$ A few numerical studies were conducted on the bifurcation routes of thermocapillary convection to chaos in rectangular liquid layers in which the Ruelle-Takens-Newhouse route was indentified..$^{35,36}$ As shown above, most studies now available are focused on the critical conditions for the onset of the oscillatory thermocapillary convection, which is the very initial regime of the bifurcation route to chaos, and the exploration of possible bifurcation routes to chaos of the thermocapillary convection is still an attractive open question due to its strong nonlinear characteristics. And most of the studies have been performed using rectangular or annular pools with relatively small lateral distance and liquids of intermediate Prandtl number (around 10). On the other hand, the thermocapillary convection and its instability are strongly dependent on the physical property of the liquid and pool geometry. Therefore, it is necessary to figure out the effects of Pr and pool geometry on the stability limits and secondary bifurcations of purely thermocapillary convection.

Recently, the researchers from National Microgravity Laboratory of Chinese Academy of Sciences are preparing a space experiment (Zona-K) on the transition to the spatiotemporal chaos of thermocapillary convection in liquid layers of finite extent under increased laterally applied temperature difference. The experiment will be conducted in the Russian module of the International Space Station (ISS) in cooperation with the Russian researchers. As the preliminary studies for the space experiment, a series of on-ground experiments are carried out based on thin liquid layers of $10 \mathrm{cSt}$ silicone oil $(\mathrm{Pr}=105.6)$ in which the thermocapillary convection is dominant even in the terrestrial conditions. As a part of the preliminary studies, the present numerical simulation aims to identify the bifurcation routes to chaos of the thermocapillary convection in liquid layers of finite extent with increasing laterally applied temperature difference. As a first step to this subject, two-dimensional (2D) liquid layers are considered in the present study and extension to more detailed three-dimensional (3D) analyses is planned as the next subject in the future. The paper is organized as follows: the section of model and method gives a brief description of the model and mathematical formulations for the present problem. In the section of Numerical Results, nonlinear dynamic behavior of the thermocapillary convection is analyzed through the portraits of the stream-function fluctuations and the free surface velocity fluctuations. Then, the bifurcation route to chaos of the thermocapillary convection is identified through the frequency power spectra, 


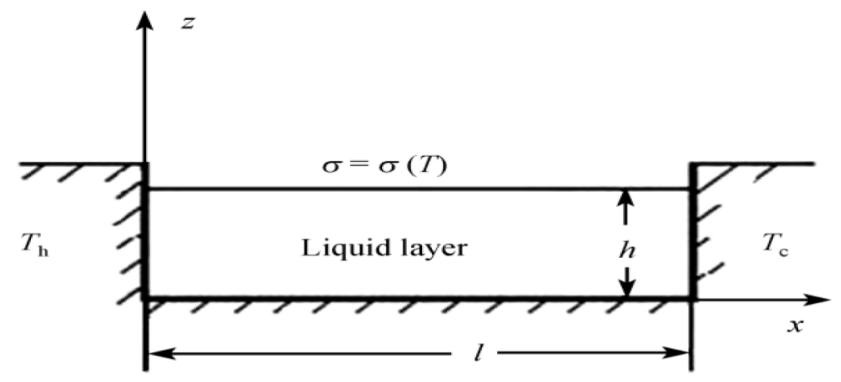

FIG. 1. Schematic of a 2D liquid layer of finite extent.

the correlation dimensions, and the trajectories in the reconstructed phase space. The conclusions are provided in Sec. IV.

\section{MODEL AND METHOD}

Note that real hydrothermal waves mostly appear as three-dimensional waves obliquely traveling toward the hot wall. However, the Prandtl number in the present study is large, and the preferred mode is a hydrothermal wave propagating almost upstream (about $10^{\circ}$ off) as predicted by Smith..$^{15}$ Moreover, the side wall effects are expected to be weak since the distance between the two side walls is relatively long (aspect ratio in the transversal direction is large). Therefore, as a first step of this project, two-dimensional (2D) liquid layers are adopted in the present numerical study. The on-ground experiments are conducted using liquid pools with different aspect ratios in the lateral direction $(\Gamma)$ ranging from 13.0 to 15.0. Present numerical simulations are conducted for pools of $\Gamma=14.25$ and 13.0, which give two representative situations of the tempo-spatial evolution of the thermocapillary convection and the corresponding sequence of bifurcations with the increasing Reynolds number.

Figure 1 shows the 2D model of liquid layer of finite extent filled with $10 \mathrm{cSt}$ silicone oil (the corresponding physical properties are shown in Table I). The liquid layer has a rigid solid at the bottom and free surface on the top, which are both assumed to be adiabatic. The temperature $T_{h}$ at the left wall is higher than $T_{c}$ at the right wall with $\Delta T=T_{h}-T_{c}$. The depth of the liquid layer is $h$, and the liquid layer extent is $l$. The liquid is assumed to be incompressible with constant viscosity and thermal diffusivity. The temperature coefficient of surface tension $\sigma_{\mathrm{T}}$ is assumed to be constant.

Present numerical simulations start with an initial condition of uniform temperature (the applied temperature difference $\Delta T$ is zero) and motion-less fluid. A semi-stepwise heating method is adopted in the present study, which is similar to the corresponding on-ground experimental studies, i.e., the temperature of the cold wall is kept at $T_{c}$ and the hot wall temperature is increased at a ramping rate of $0.05 \mathrm{~K} / \mathrm{s}$ for a time span of $1 \mathrm{~s}$, which results in the applied temperature difference $\Delta T=0.05 \mathrm{~K}$. Then, the applied temperature difference is kept constant over a time span more than $5000 \mathrm{~s}$, which is long enough to allow the full development of the thermocapillary convection under the assigned $\Delta T$. Then, the hot wall temperature is further raised at the same ramping rate of $0.05 \mathrm{~K} / \mathrm{s}$ for a time span of $1 \mathrm{~s}$ and then $\Delta T=0.10 \mathrm{~K}$ is kept over a time span more than $5000 \mathrm{~s}$ once again. By repeating the same process of "semi-stepwise increase of $\Delta T$ followed by a long enough time span with the constant $\Delta T$," the process toward the chaos is investigated.

In the present study, vorticity $\omega$ and stream-function $\psi$ are, respectively, introduced as

$$
\nabla \times \psi^{*}=\mathbf{V}^{*}, \quad \nabla \times \nabla \times \psi^{*}=\omega^{*},
$$

TABLE I. Physical properties of $10 \mathrm{cSt}$ silicone oil.

\begin{tabular}{lcccccc}
\hline \hline$\rho\left(\mathrm{kg} / \mathrm{m}^{3}\right)$ & $\nu\left(\mathrm{m}^{2} / \mathrm{s}\right)$ & $\mu(\mathrm{kg} / \mathrm{ms})$ & $\alpha\left(\mathrm{m}^{2} / \mathrm{s}\right)$ & $\sigma(\mathrm{N} / \mathrm{m})$ & $\sigma_{T}(\mathrm{~N} / \mathrm{mK})$ & $\operatorname{Pr}$ \\
\hline 935 & $10^{-5}$ & $9.35 \times 10^{-3}$ & $9.47 \times 10^{-8}$ & $2.01 \times 10^{-2}$ & $-6.12 \times 10^{-5}$ & 105.6 \\
\hline \hline
\end{tabular}


TABLE II. Physical scales.

\begin{tabular}{lcccccccc}
\hline \hline Variable & $x, z$ & $u, w$ & $p$ & $T-T_{c}(\mathrm{~K})$ & $\mathrm{t}$ & $\omega$ & $\psi$ & $f$ \\
\hline Scale & $h$ & $U=\left|\sigma_{T}\right| \Delta T / \mu$ & $\left|\sigma_{T}\right| \Delta T / h$ & $\Delta T$ & $h^{2} / v$ & $U / h$ & $U h$ & $v / h^{2}$ \\
\hline \hline
\end{tabular}

where $\mathbf{V}^{*}\left(u^{*}, w^{*}\right)$ is the flow velocity with the components $u^{*}$ and $w^{*}$ along the horizontal direction $x^{*}$ and the vertical direction $z^{*}$, respectively. Then, the dimensionless governing equations can be expressed as follows:

$$
\begin{gathered}
\frac{\partial \omega^{*}}{\partial t^{*}}+\operatorname{ReV}^{*} \cdot \nabla \omega^{*}=\Delta \omega^{*} \\
\operatorname{Pr} \frac{\partial T^{*}}{\partial t^{*}}+M a \mathbf{V}^{*} \cdot \nabla T^{*}=\Delta T^{*},
\end{gathered}
$$

where the asterisk $\left(^{*}\right)$ is for the dimensionless values. Note that the equation of mass conservation is automatically satisfied. The corresponding dimensionless numbers are defined as $\operatorname{Pr}=v / \alpha$, $\operatorname{Re}=\left|\sigma_{T}\right| \Delta T h / \mu \nu, M a=\operatorname{Re} \times \operatorname{Pr}=\left|\sigma_{T}\right| \Delta T h / \mu \alpha, \Gamma=l / h$. Here, $\operatorname{Pr}$ is the Prandtl number, $M a$ the Marangoni number, $\Gamma$ the aspect ratio, and the scales for the physical quantities are given in Table II.

The dimensionless boundary conditions are as follows:

$$
\begin{aligned}
& x^{*}=0: \psi^{*}=0, \omega^{*}=-\frac{\partial^{2} \psi^{*}}{\partial x^{* 2}}, T^{*}=1, \\
& x^{*}=\Gamma: \psi^{*}=0, \omega^{*}=-\frac{\partial^{2} \psi^{*}}{\partial x^{* 2}}, T^{*}=0, \\
& z^{*}=0: \psi^{*}=0, \omega^{*}=-\frac{\partial^{2} \psi^{*}}{\partial z^{* 2}}, \frac{\partial T^{*}}{\partial z^{*}}=0 .
\end{aligned}
$$

The free surface is considered as a plane in initial quiescent liquid layer. The deformation of the free surface is negligible in the present study since it is so small compared to the height of the liquid layer of $10 \mathrm{cSt}$ silicone oil. ${ }^{37}$ Therefore, the free surface is located at $z^{*}=1$. The vorticity at free surface is determined from the equilibrium of tangential stress, and the boundary condition at the free surface is

$$
\psi^{*}=0, \quad \omega^{*}=\frac{\partial T^{*}}{\partial x^{*}}, \quad \frac{\partial T^{*}}{\partial z^{*}}=0 .
$$

A hybrid finite element method of fractional steps ${ }^{38}$ is adopted in the present study. The number of grids is $121 \times 21$ in the directions of $x$ and $z$, respectively, and the computation domain is divided into 4800 irregular triangular elements with 2541 nodes. The details of the numerical method and code validation can be found elsewhere ${ }^{39}$ and the non-dimensional time step 0.0001 for the calculation is adopted. To identify the different states in the bifurcation route to chaos of the thermocapillary convection, the fast Fourier transform is adopted on the time history of the dynamic variables at the monitoring points. The periodic oscillation, quasi-periodic oscillation, and period-doubling scenarios can be recognized from the corresponding FFT power spectrum. Chaotic convection is considered to occur when the signal spectrum develops into broadband. Moreover, the time history of the dynamic variable can be represented in the reconstructed phase spaces of a train of embedding dimensions. Then, the final embedding dimension and the correlation dimension can be obtained through G-P algorithm. ${ }^{40}$ In this way, the nonlinear dynamic characteristic of the thermocapillary convection in the $2 \mathrm{D}$ liquid layers of finite extent can be explicitly described.

\section{NUMERICAL RESULTS}

The thermocapillary convection is steady at a small Reynolds number and transfers to oscillatory convection when the Reynolds number exceeds a critical value $\operatorname{Re}_{c}$ (the critical Reynolds number for the onset of hydrothermal wave instability). At further increased Reynolds number, the 
thermocapillary convection evolves through various complex ways (depending on the parameters $\operatorname{Pr}$ and $\Gamma$ ) into chaotic flow. The over-critical-parameter from the transition point $\operatorname{Re}_{c}$ is described as $\varepsilon=\left(\operatorname{Re}-\operatorname{Re}_{c}\right) / \operatorname{Re}_{c}$. Note that non-dimensional numerical simulations are carried out in the present study, and the results are described in both non-dimensional and dimensional way for the future direct comparison with the on-ground experimental results.

For the liquid layer of aspect ratio 14.25 , the steady thermocapillary convection ends up at the critical temperature difference $\Delta T_{c}=18.80 \mathrm{~K}\left(\operatorname{Re}_{c}=49\right)$. When the thermocapillary convection get unsteady as $\Delta T$ goes beyond the critical value, the fluctuation is defined as the difference between the dynamic variable and its time-averaged value, e.g., $\psi(x, z, t)=\psi^{\prime}(x, z, t)+\psi_{0}(x, z)$, where $\psi^{\prime}(x, z, t)$ is the stream-function fluctuation and $\psi_{0}(x, z)=\frac{1}{\tau} \int_{0}^{\tau} \psi(x, z, t) d t$ is the corresponding time-averaged stream-function, $\tau$ is the time length much larger than the oscillation period. The numerical results show that the strength of the fluctuation $\left(\psi^{\prime}\right)$ is at least two orders less than the time-averaged value $\left(\psi_{0}\right)$. Therefore, the portraits of the stream-functions qualitatively keep the general trend of the unicellular structure with increasing applied temperature difference, i.e., the temperature gradient on the free surface drives the fluid from the hot wall to the cold wall, while the liquid at the bottom of the liquid layer flows from the cold wall to the hot wall.

At $\operatorname{Re}_{c}=49\left(\Delta T_{c}=18.80 \mathrm{~K}\right)$, as shown in Fig. 2(a), two pairs of stream-function fluctuation cells arise and occupy the partial region of the liquid layer near the hot region. In the following plots, solid line corresponds to a positive stream function fluctuation while dashed line to a negative one. As the Reynolds number increases, the number of the fluctuation cells increases. The fluctuation cells become more rectangular with similar sizes and distribute regularly in the central region of the liquid layer. The fluctuation cells near the hot and cold walls are relatively small and weak. When the Reynolds number is increased up to $\operatorname{Re}_{c}=105, \varepsilon=1.14$ ( $\left.\Delta T=40.00 \mathrm{~K}\right)$, as shown in Fig. 2(b), the limited extent of the liquid layer does not allow further increase of the fluctuation cell number and the boundary effect becomes significant. The fluctuation cells become irregular with different size and strength. Further increase of the Reynolds number to $\mathrm{Re}=157, \varepsilon=2.20(\Delta T=60.00 \mathrm{~K})$, as shown in Fig. 2(c), the fluctuation cells become very irregular. Figure 3 shows the SpatioTemporal-Diagram

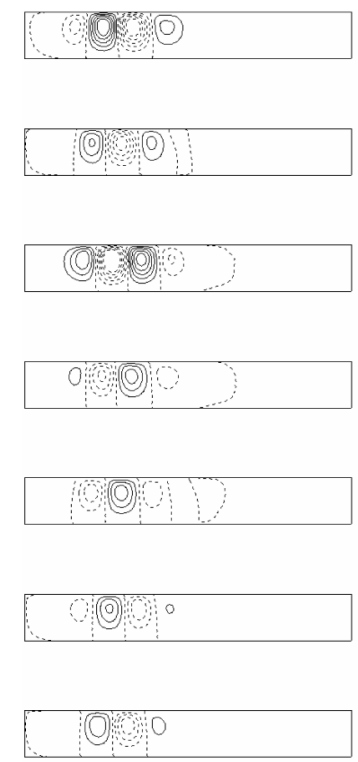

(a)

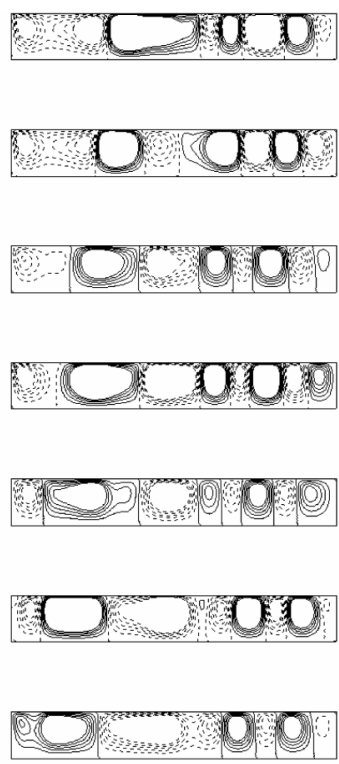

(b)

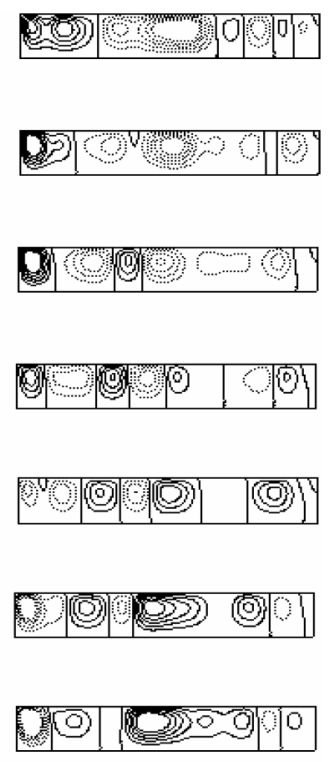

(c)

FIG. 2. Time evolutions of the stream-function fluctuations $\psi^{\prime}$ (right) of the thermocapillary convection in liquid layer of aspect ratio 14.25 at different instants: (a) $\Delta T=18.80 \mathrm{~K}\left(\operatorname{Re}=\operatorname{Re}_{c}=49\right)$ from $5010 \mathrm{~s}$ (top) to $5034 \mathrm{~s}$ (bottom) at an interval $4 \mathrm{~s}$, (b) $\Delta T=40.00 \mathrm{~K}$ ( $\operatorname{Re}=105, \varepsilon=1.13$ ) from $5010 \mathrm{~s}$ (top) to $5022 \mathrm{~s}$ (bottom) at an interval $2 \mathrm{~s}$, (c) $\Delta T=60.00 \mathrm{~K}$ ( $\operatorname{Re}=157$, $\varepsilon=2.19$ ) from $5000 \mathrm{~s}$ (top) to $5036 \mathrm{~s}$ (bottom) at an interval $6 \mathrm{~s}$. The stream-function fluctuation is positive for the solid lines in the plots while negative for the dashed lines. 


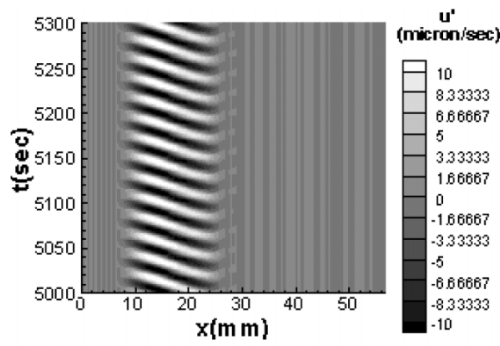

(a)

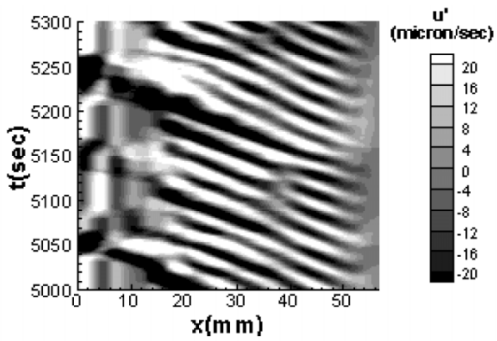

(e)
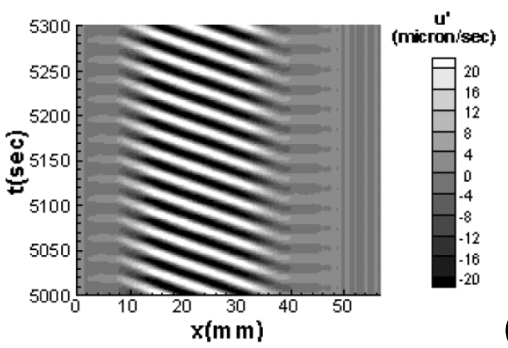

(b)
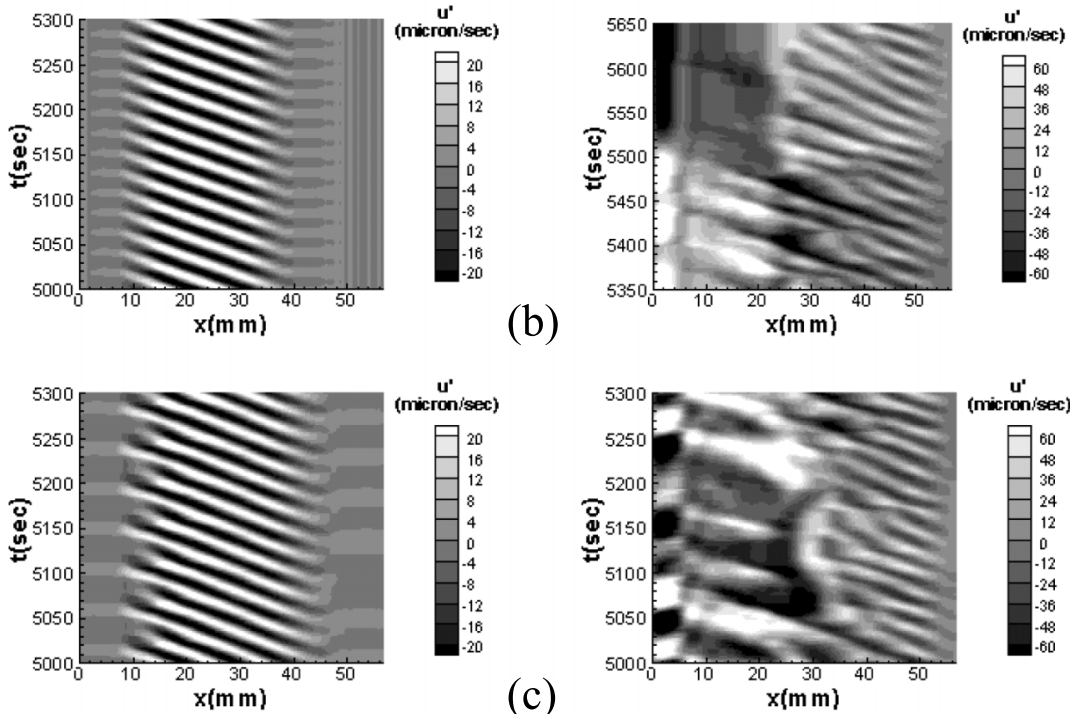

(c)

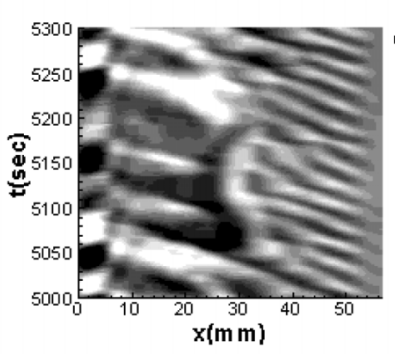

(f)
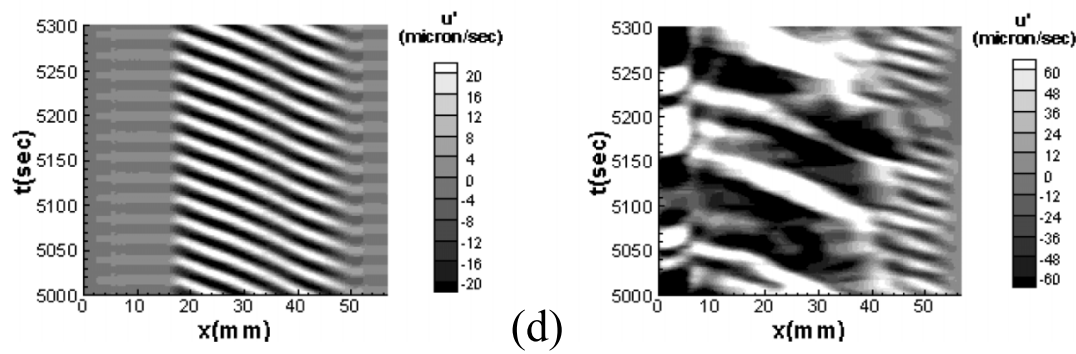

(g)

(d)

FIG. 3. Spatial-temporal diagram of surface velocity fluctuation $\mathrm{u}^{\prime}(\mathrm{x}, \mathrm{t})$ with various temperature differences (Reynolds numbers): (a) $\Delta T=18.80 \mathrm{~K}\left(\operatorname{Re}=\operatorname{Re}_{c}=49\right)$, (b) $\Delta T=22.00 \mathrm{~K}(\operatorname{Re}=58, \varepsilon=0.17)$, (c) $\Delta T=24.50 \mathrm{~K}(\operatorname{Re}=64, \varepsilon=0.30)$, (d) $\Delta T=28.65 \mathrm{~K}(\mathrm{Re}=75, \varepsilon=0.52)$, (e) $\Delta T=33.00 \mathrm{~K}(\mathrm{Re}=86, \varepsilon=0.76)$, (f) $\Delta T=40.00 \mathrm{~K}(\mathrm{Re}=105, \varepsilon=1.13)$, (g) $\Delta T=50.00 \mathrm{~K}(\mathrm{Re}=131, \varepsilon=1.66),(\mathrm{h}) \Delta T=60.00 \mathrm{~K}(\mathrm{Re}=157, \varepsilon=2.19)$. The diagrams indicate the propagation of wavy propagation of the surface velocity fluctuations.

(STD) of the surface velocity fluctuation $u^{\prime}(x, t)$ at different Reynolds numbers (applied temperature differences). When the Reynolds number is relatively small $\operatorname{Re}<80, \varepsilon<0.63(\Delta T<30.00 \mathrm{~K})$, as shown in Figs. 3(a)-3(d), the strong velocity fluctuation appears in the central region of the liquid layer, while the fluctuation near the hot and cold walls is relatively weak, and the region with strong fluctuation spreads with the increasing Reynolds number. It can be clearly seen that the fluctuation arises in the cold region and propagates toward the hot region. This structure keeps until it reaches the hot wall and gets weakened. Therefore, the fluctuation shows a typical feature of hydrothermal waves as expected by the stability analysis of Smith and Davis ${ }^{15}$ in the central region of the liquid layer and is weakened in the regions close to the hot and cold walls. When the Reynolds number is further increased, as shown in Figs. 3(e)-3(f), the fluctuation gets stronger in the whole liquid layer. Near the hot wall, the fluctuation shows a complex spatiotemporal evolution without traveling wave. In the other region, relatively regularly structured traveling fluctuation appears with fluctuating period. At 

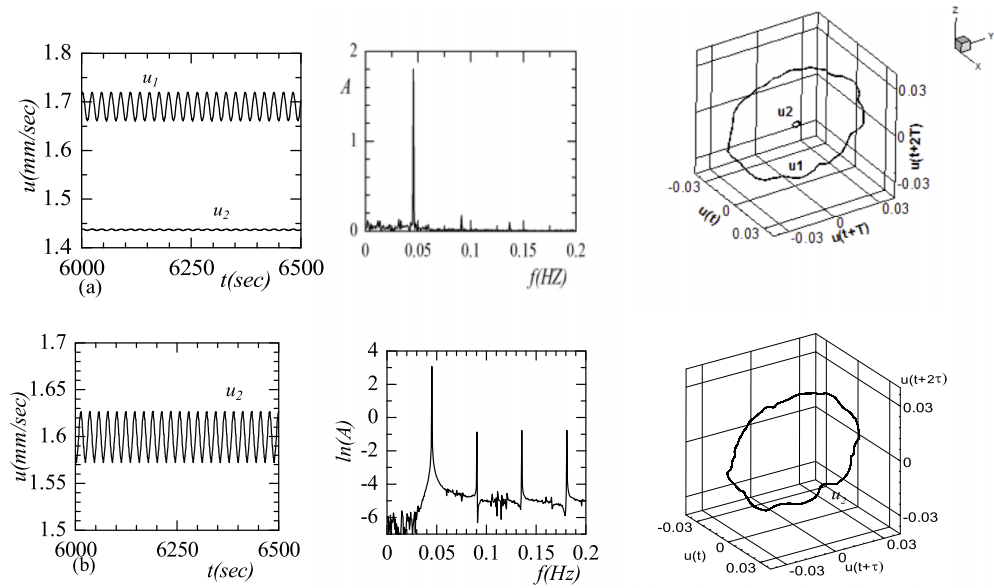

(a)
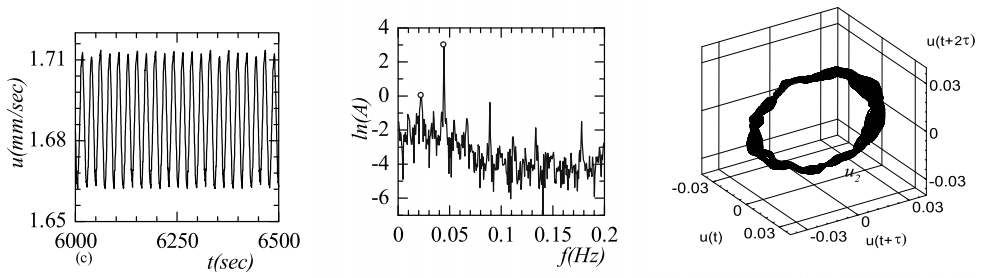

(b)
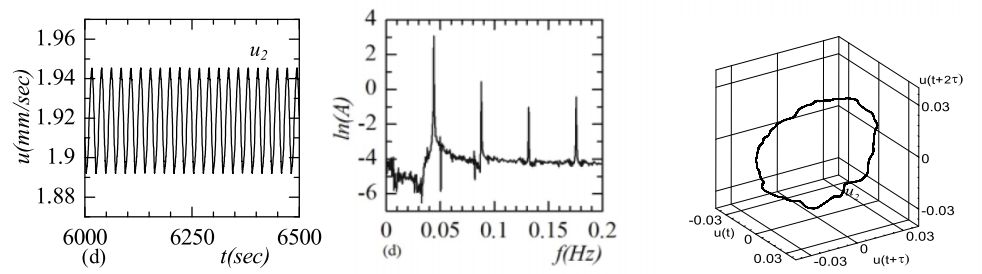

(c)
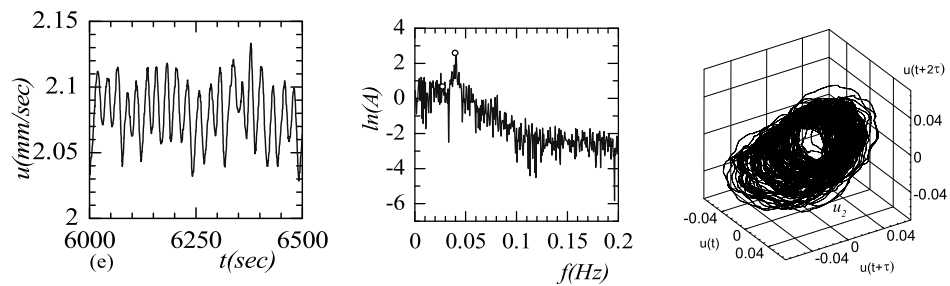

(d)
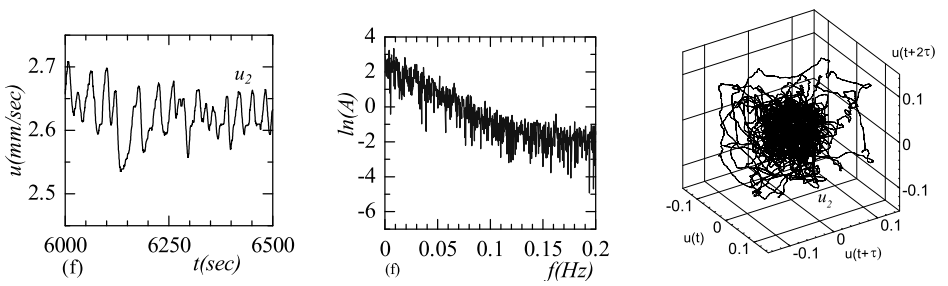

(e)

FIG. 4. Time histories, power spectra, and phase trajectories of the surface velocity fluctuations in liquid layer of aspect ratio 14.25 with various temperature differences (Reynolds numbers): (a) $\Delta T=18.80 \mathrm{~K}\left(\operatorname{Re}=\operatorname{Re}_{c}=49\right.$ ), (b) $\Delta T=22.00 \mathrm{~K}$ ( $\operatorname{Re}=58, \varepsilon=0.17),(\mathrm{c}) \Delta T=24.50 \mathrm{~K}(\operatorname{Re}=64, \varepsilon=0.30),(\mathrm{d}) \Delta T=28.65 \mathrm{~K}(\operatorname{Re}=75, \varepsilon=0.52),(\mathrm{e}) \Delta T=33.00 \mathrm{~K}(\operatorname{Re}=86$, $\varepsilon=0.76)$, (f) $\Delta T=60.00 \mathrm{~K}(\operatorname{Re}=157, \varepsilon=2.19)$.

last, when the Reynolds number is increased up to $\operatorname{Re}>157, \varepsilon>2.20(\Delta T>60.00 \mathrm{~K})$, the region occupied by the irregular fluctuation spreads over the whole liquid layer as shown in Fig. 3(h).

Time histories of the free surface velocities are recorded at two monitoring points, $x_{1}=$ $14.25 \mathrm{~mm}\left(x_{1}^{*}=\Gamma / 4\right)$ and $x_{2}=28.5 \mathrm{~mm}\left(x_{2}^{*}=\Gamma / 2\right)$. The free surface velocity fluctuation $u^{\prime}\left(x_{2}, h, t\right)$ 
TABLE III. Parameters selected for the reconstructed phase space and the correlation dimensions calculated with $u_{1}$ or $u_{2}$ at various temperature differences.

\begin{tabular}{lcccccc}
\hline \hline$\Delta \mathrm{T}(\mathrm{K})$ & 18.80 & 22.00 & 24.50 & 28.65 & 33.00 & 60.00 \\
\hline Fluctuation velocity & $u_{1}$ & $u_{2}$ & $u_{2}$ & $u_{2}$ & $u_{2}$ & $u_{2}$ \\
Embedding dimension & 3 & 3 & 3 & 3 & 3 & 6 \\
Time delay $\tau$ & 16 & 16 & 17 & 17 & 11 & 15 \\
Correlation dimension & 1.0 & 1.0 & 1.1 & 1.0 & 1.5 & 3.3 \\
\hline \hline
\end{tabular}

(noted as $u_{2}$ hereafter) is adopted to investigate the nonlinear dynamic characteristics of the thermocapillary convection. Such a selection is to minimize the boundary effects of the limited liquid layer. With various Reynolds numbers, Fig. 4 shows the sequence of the sample signals of $u_{2}$ with corresponding power spectra and the projections of the trajectories in the reconstructed phase space. The parameters selected for the phase space reconstruction and the correlation dimensions obtained are listed in Table III. At the critical Reynolds number $\operatorname{Re}_{c}=49\left(\Delta T_{c}=18.80 \mathrm{~K}\right), u_{1}=u^{\prime}\left(x_{1}, h, t\right)$ is also studied as it is much larger than $u_{2}$. A fundamental frequency $f_{1}=0.046 \mathrm{~Hz}$ can be clearly identified in the power spectrum. The correlation dimension is 1.0 and the corresponding phase trajectory is a typical limit cycle. The scenarios demonstrate that the steady thermocapillary convection ends up and shifts to periodic oscillatory convection via Hopf bifurcation. This periodic oscillatory state remains till $\operatorname{Re}=60, \varepsilon=0.22(\Delta T=23.00 \mathrm{~K})$, and the fundamental frequency $f_{1}$ slightly decreases with the increasing $\Delta T$. The periodic oscillatory state also emerges in the range of $\operatorname{Re}=73-79, \varepsilon=0.49-0.61(\Delta T=28.00 \mathrm{~K}-30.00 \mathrm{~K})$ with the significant development of the harmonics of the fundamental frequency. Besides the periodic oscillatory states, the power spectra for the other temperature differences are relatively complex. Some typical cases are analyzed in Fig. 4. At $\operatorname{Re}=64, \varepsilon=0.31(\Delta T=24.50 \mathrm{~K})$, the signal broadband develops; however, the fundamental frequency $f_{1}=0.045 \mathrm{~Hz}$ still can be identified and a second fundamental frequency $f_{2}=0.019 \mathrm{~Hz}$ appears (peaks of the fundamental frequencies are marked by a circle in the plot). Other peaks can be expressed by linear combination of two incommensurate fundamental frequencies. This is a typical quasi-periodic oscillatory state of the thermocapillary convection. The corresponding correlation dimension is 1.1 and the trajectory in the reconstructed phase space shows the form of a circle "cluster" formed by a single closed line. At $\operatorname{Re}=86, \varepsilon=0.76(\Delta T=33.00 \mathrm{~K})$, the number of the peaks in the power spectrum increases and some locally broadened peaks appear due to the overlap of the peaks. However, two incommensurate fundamental frequencies, $f_{1}$ and $f_{2}$, still can be identified, and all other peaks can be expressed by the linear combination of these two incommensurate fundamental frequencies. Therefore, the thermocapillary convection is still in the quasi-periodic oscillatory state. The corresponding correlation dimension is 1.5 and the trajectory in the reconstructed phase space also shows the form of a closed circle "cluster" but with more random spatial distribution compared to the case of $\operatorname{Re}=64, \varepsilon=0.31(\Delta T=24.50 \mathrm{~K})$. The power spectrum at $\operatorname{Re}=157, \varepsilon=2.20(\Delta T=60.00 \mathrm{~K})$ finally develops to a state of broadband without any fundamental frequency, and the corresponding trajectory in the reconstructed phase space is random. The correlation dimension is 3.3 significantly larger than the other cases in Table III. This sudden increase of the correlation dimension implies a transition to a different state. On the other hand, the existence of the correlation dimension distinguishes the present state from the signal random. To verify that the state is chaotic, the largest Lyapunov exponents for selected values of the bifurcation parameter are calculated. The results demonstrate a good convergence of the algorithm and reveal the positive value of 0.057 . This proves that for this value of the forcing parameter, the state is chaotic. The fundamental frequencies indentified in the power spectra with various Reynolds numbers are summarized in Fig. 5. It can be seen that the fundamental frequency $f_{1}$ slightly decreases with the increasing $\operatorname{Re}(\Delta T)$ in the range of $\operatorname{Re}<79, \varepsilon<0.61(\Delta T<30.00 \mathrm{~K})$ and then shows a relatively quick decrease in the range of $\operatorname{Re}=79-105, \varepsilon=0.61-1.14(\Delta T=30.00-40.00 \mathrm{~K})$. The fundamental frequency cannot be indentified in the range of $\operatorname{Re}=105-183, \varepsilon=1.14-2.73$ $(\Delta T=40.00-70.00 \mathrm{~K})$. On the other hand, the second fundamental frequency $f_{2}$ only emerges in 


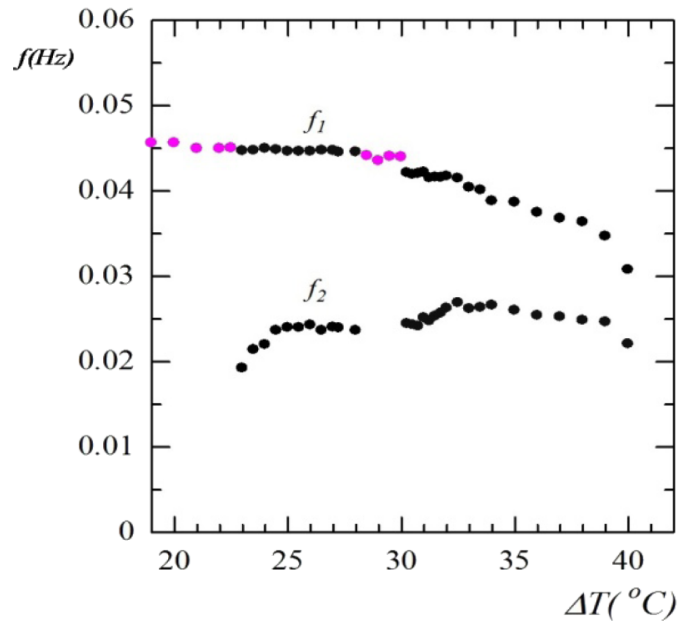

(a)

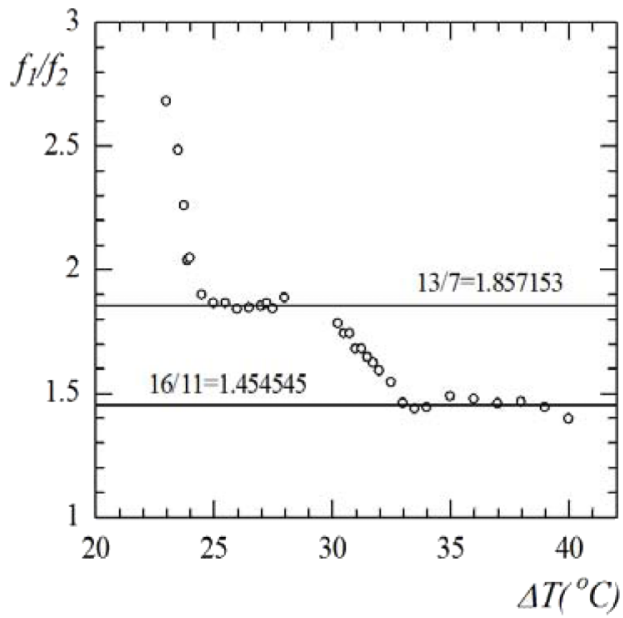

(b)

FIG. 5. (a) Two fundamental frequencies $\left(f_{1}\right.$ and $\left.f_{2}\right)$ and (b) their ratios of the oscillatory thermocapillary convection in liquid layer of aspect ratio 14.25 with various temperature differences. The colored dots are the fundamental frequencies for the periodic oscillatory state.

the periodic oscillatory states in the range of $\operatorname{Re}=60-73, \varepsilon=0.22-0.49(\Delta T=23.00-28.00 \mathrm{~K})$ and $\operatorname{Re}=79-105, \varepsilon=0.61-1.14(\Delta T=30.00-40.00 \mathrm{~K})$, as shown in Fig. 5(a). The ratio $f_{1} / f_{2}$ plotted in Fig. 5(b) reveals that the frequency-locking emerges in both quasi-periodic oscillatory states at $f_{1} / f_{2}=13 / 7=1.8571$ and $f_{1} / f_{2}=16 / 11=1.4546$, respectively. Note that the emergence of frequency-locking or a third incommensurate fundamental frequency is necessary for the shift from quasi-periodic oscillatory state to chaos.

A complete overview on all bifurcation sequence identified in the present case ( $\operatorname{Pr}=105.6, \Gamma=$ $14.25)$ is as follows: steady state $(\operatorname{Re}<49, \Delta T<18.80 \mathrm{~K}) \rightarrow$ periodic oscillatory state

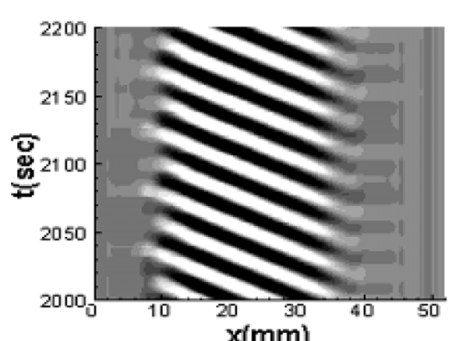

(a)

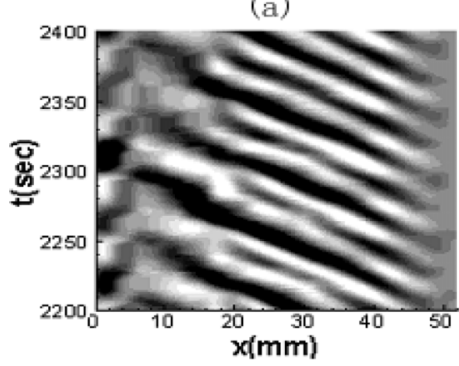

(c)
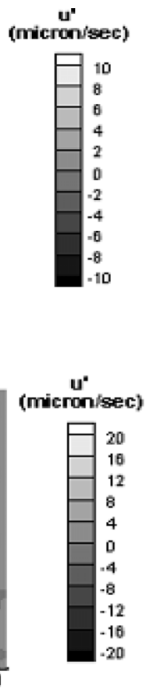

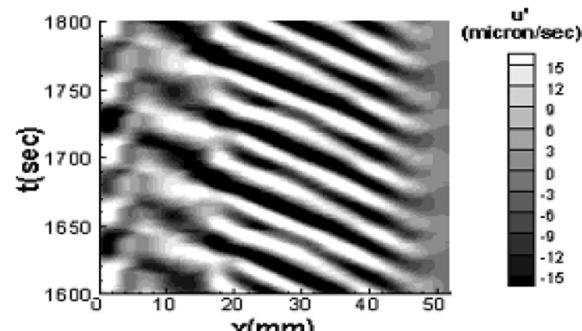

(b)

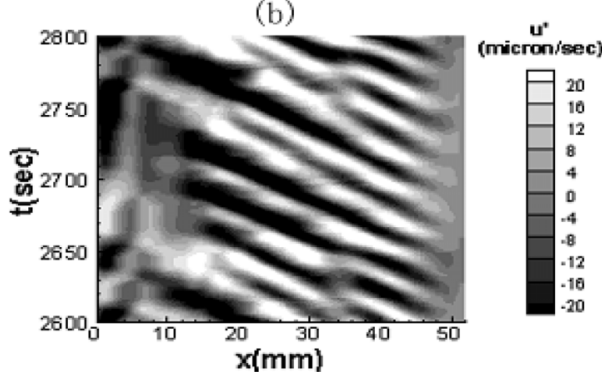

(d)

FIG. 6. Spatial-temporal diagram of surface velocity fluctuation $\mathrm{u}^{\prime}(\mathrm{x}, \mathrm{t})$ with various temperature differences (Reynolds numbers): (a) $\Delta T=21.02 \mathrm{~K}\left(\operatorname{Re}=\operatorname{Re}_{c}=55\right)$, (b) $\Delta T=30.20 \mathrm{~K}(\operatorname{Re}=79, \varepsilon=0.44)$, (c) $\Delta T=32.10 \mathrm{~K}(\operatorname{Re}=84, \varepsilon=0.53)$, (d) $\Delta T=35.00 \mathrm{~K}(\mathrm{Re}=92, \varepsilon=0.67)$. The diagrams indicate the propagation of wavy propagation of the surface velocity fluctuations. 

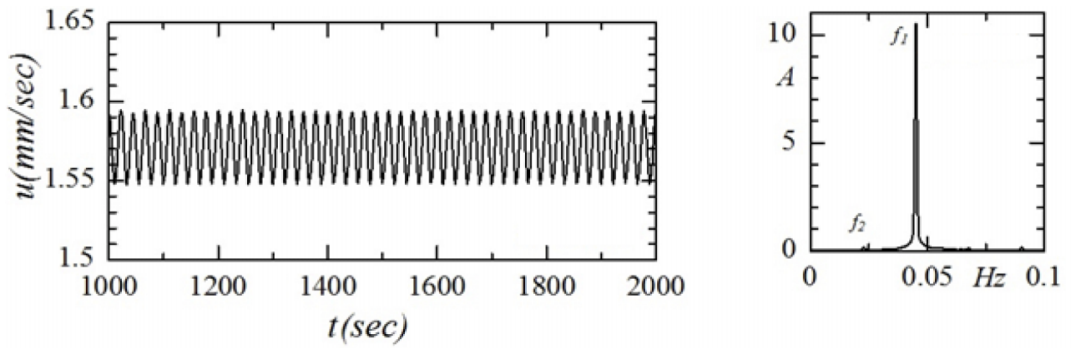

(a)
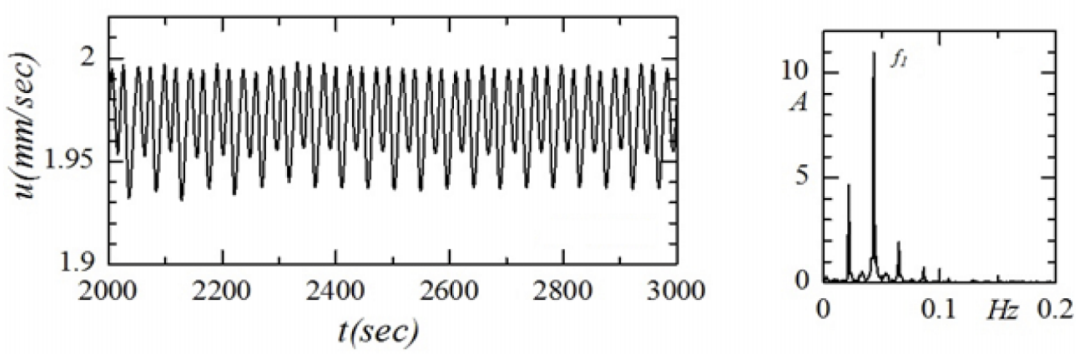

(b)
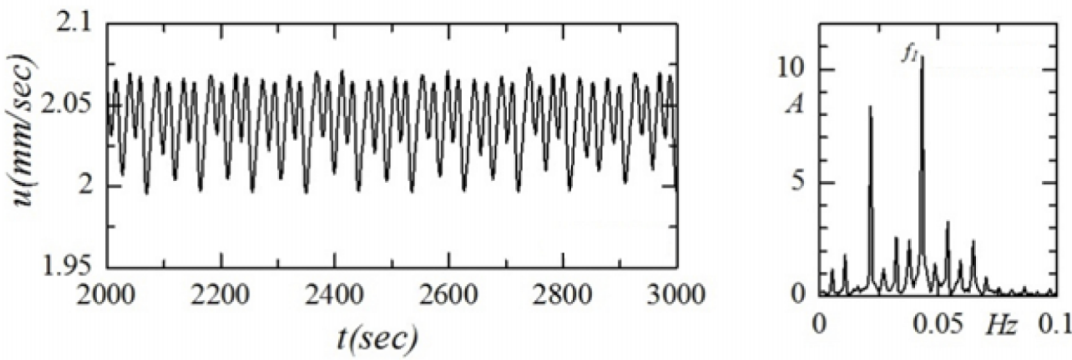

(c)
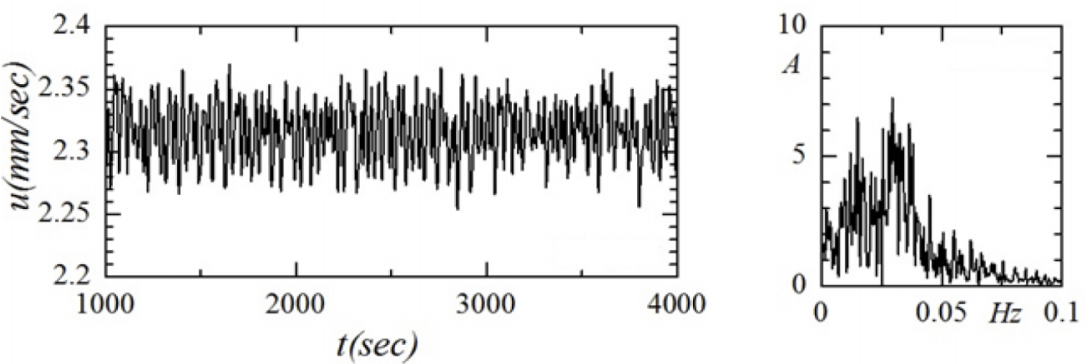

(d)

FIG. 7. Time histories of the free surface velocity and corresponding power spectra at $x=26.0 \mathrm{~mm}(0.5 \Gamma)$ in liquid layer of aspect ratio 13.0 at with various temperature differences (Reynolds numbers): (a) $\Delta T=21.02 \mathrm{~K}\left(\operatorname{Re}=\operatorname{Re}_{c}=55\right)$, (b) $\Delta T=30.20 \mathrm{~K}(\operatorname{Re}=79, \varepsilon=0.44),(\mathrm{c}) \Delta T=32.10 \mathrm{~K}(\operatorname{Re}=84, \varepsilon=0.53),(\mathrm{d}) \Delta T=35.00 \mathrm{~K}(\operatorname{Re}=92, \varepsilon=0.67)$.

$(\operatorname{Re}=49-60, \varepsilon=0-0.22, \Delta T=18.80-23.00 \mathrm{~K}) \rightarrow$ quasi-periodic oscillatory state with frequencylocking $(\operatorname{Re}=60-73, \varepsilon=0.22-0.49, \Delta T=23.00-28.00 \mathrm{~K}) \rightarrow$ periodic oscillatory state $(\operatorname{Re}=73-79$, $\varepsilon=0.49-0.61, \Delta T=28.00-30.00 \mathrm{~K}) \rightarrow$ quasi-periodic oscillatory state with frequency-locking $(\operatorname{Re}=79-105, \varepsilon=0.61-1.14, \Delta T=30.00-40.00 \mathrm{~K}) \rightarrow$ chaos $(\operatorname{Re}=105-183, \varepsilon=1.14-2.73, \Delta T=$ 40.00-70.00 K). Note that the Reynolds number ranges are approximately divided and the intermediate state may exist between neighboring oscillatory states.

For the liquid layer of aspect ratio 13.0, the steady thermocapillary convection ends up at the critical Reynolds number $\operatorname{Re}_{c}=55(\Delta T=21.02 \mathrm{~K})$ and periodic oscillatory thermocapillary convection arises. Similar to the case of the liquid layer of aspect ratio 14.25 , the surface velocity fluctuation $u^{\prime}(x, t)$ arises at the partial region near the hot wall, while the fluctuation in the other region is very weak as shown in Fig. 6(a). However, the region occupied by the fluctuation is much wider than the case of the liquid layer of aspect ratio 14.25 due to the emergence of three 
pairs of stream-function fluctuation cells with similar rectangular shape. As shown in Fig. 6(a), the fluctuation propagates from the cold region toward the hot region, which shows the typical feature of hydrothermal wave. With the increasing Reynolds number (applied temperature difference), the surface velocity fluctuation $u^{\prime}(x, t)$ gets strong and spreads over the whole liquid layer, and the fluctuation near the hot wall becomes complex with irregular spatiotemporal evolution, while the fluctuation in the other region keeps the pattern of hydrothermal wave as shown in Figs. 6(b)6(d). When the Reynolds number is further increased up to $\operatorname{Re}=105, \varepsilon=0.91(\Delta T=40.00 \mathrm{~K})$, the fluctuation in the whole region gets complex and irregular and the flow becomes chaotic.

The time history of the free-surface velocity at $x^{*}=\Gamma / 2(x=26.0 \mathrm{~mm})$ is adopted to investigate the nonlinear dynamic characteristics of the thermocapillary convection. Figure 7 shows the sequence of the sample signals and the corresponding power spectra with various Reynolds numbers (applied temperature differences). At the critical condition for the onset of hydrothermal waves $\operatorname{Re}_{c}=55\left(\Delta T_{c}=21.02 \mathrm{~K}\right)$, the periodic oscillatory thermocapillary convection arises in the liquid layer, and the major frequency $f_{1}=0.04512 \mathrm{~Hz}$ relating to the highest peak can be clearly identified in the power spectrum. Note that the peak at its sub-harmonic frequency $f_{2}=f_{1} / 2$ emerges in the power spectrum simultaneously. With the further increase of the Reynolds number, the peaks at $f_{1} / 4$ with its harmonics and the peaks at $f_{1} / 8$ and its harmonics emerge in the power spectrum at $\operatorname{Re}_{1}=79, \varepsilon=0.44\left(\Delta T_{1}=30.20 \mathrm{~K}\right)$ and $\operatorname{Re}_{2}=84, \varepsilon=0.53\left(\Delta T_{2}=32.10 \mathrm{~K}\right)$ successively. When the Reynolds number is further increased to $\operatorname{Re}=105, \varepsilon=0.91(\Delta T=40.00 \mathrm{~K})$, the power spectrum develops into the signal broadband without any fundamental frequency, and the flow becomes chaotic. For the present case, the typical period-doubling bifurcation route can be clearly identified from the power spectra, and the investigations on the trajectories in the re-constructed phase space are ignored. In summary, the steady state of the thermocapillary convection in the present case undergoes period-doubling cascades leading to chaos. The Feigenbaum constant for the present case is calculated as $\frac{\mathrm{Re}_{1}-\mathrm{Re}_{c}}{\mathrm{Re}_{2}-\mathrm{Re}_{1}}=\frac{79-55}{84-79}=4.8$ with the relative error 3.0\% compared to the theoretical value of $4.66420 .^{3}$

\section{CONCLUSIONS}

As a part of preliminary studies for the future space experiment (Zona-K) in the Russian module of the International Space Station (ISS), thermocapillary convection in two-dimensional liquid layers of $10 \mathrm{cSt}$ silicone oil was numerically investigated in the present study. The results show that the onset of oscillatory convection is accompanied by the fluctuations of dynamic variables and the propagation of small perturbation roll cells just like the hydrothermal waves in an infinite liquid layer predicted by Smith and Davis. ${ }^{18}$ On the other hand, the finite extent of the liquid layer significantly influences the development process of the thermocapillary convection at highly super critical states. The secondary instabilities of the hydrothermal waves are caused by local changes of surface temperature gradient, which may be caused by merging of neighboring roll cells or splitting of roll cells. Considering the number and size of the roll cells is limited by the aspect ratio of the liquid layer, secondary instabilities may be influenced not only by the Reynolds number (the applied over-all temperature difference) but also by the aspect ratio. The present results show that in a liquid layer of aspect ratio 14.25, with the increasing Reynolds number, the steady thermocapillary convection experiences two consecutive transitions from periodic oscillatory state to quasi-periodic oscillatory state with frequency-locking before emergence of chaotic flow. In the liquid layer of aspect ratio 13.0, the steady thermocapillary convection undergoes period-doubling cascades leading to chaotic flow. These results clearly indicate that the bifurcation route of the thermocapillary convection changes very sensitively by the aspect ratio of the liquid layer.

\section{ACKNOWLEDGMENTS}

This work was supported by the National Natural Science Foundation of China (Grant No. 11032100). 
${ }^{1}$ H. Poincare, "Les methods nouvelles de la mechanique Celeste, Gauthier-Villars, Paris," in NASA Translation TT, F-405/452 (US Fed Clearinghouse, Springfield, VA, USA, 1967), (in English).

2 E. N. Lorentz, "Deterministic nonperiodic flow," J. Atmos. Sci. 20, 130 (1963).

${ }^{3}$ M. J. Feigenbaum, "The onset spectrum of turbulence," Phys. Lett. A 74, 375 (1979).

${ }^{4}$ J. P. Gollub and S. V. Benson, "Many routes to turbulent convection," J. Fluid Mech. 100, 449 (1980).

${ }^{5}$ R. Braun, F. Feudel, and P. Guzdar, "Route to chaos for a two-dimensional externally driven flow," Phys. Rev. E 58, 1927 (1998).

${ }^{6}$ E. Bucchignani and F. Stella, “Transitions to chaos," Numer. Heat Transfer, Part A 36, 17 (1999).

${ }^{7}$ A. K. Saha, G. Biswas, and K. Muralidhar, "Transition and chaos in two-dimensional flow past a square cylinder," J. Eng. Mech. 126, 523 (2000).

${ }^{8}$ S. Libchaber and J. Maurer, “Local probe in a Rayleigh-Benard experiment in liquid helium,” J. Phys. Lett. 39, 369 (1978).

${ }^{9}$ D. Ruelle and F. Takens, "On the nature of turbulence," Commun. Math. Phys. 20, 167 (1971).

${ }^{10}$ S. Newhouse, D. Ruelle, and F. Takens, "Occurrence of strange axiom-A attractors near quasi periodic flows on Tm, $\mathrm{m} \geq 3$," Commun. Math. Phys. 64, 35 (1978).

${ }^{11}$ Y. Pomeau and P. Manneville, "Intermittent transition to turbulence in dissipative dynamical systems," Commun. Math. Phys. 74, 189 (1980).

12 S. H. Davis, "Thermocapillary instabilities," Annu. Rev. Fluid Mech. 19, 403 (1987).

${ }^{13}$ M. F. Schatz and G. P. Neitzel, "Experiments on thermocapillary instability," Annu. Rev. Fluid Mech. 33, 93 (2001).

${ }^{14}$ W. R. Hu, Z. M. Tang, and K. Li, "Thermocapillary convection in floating zone," Appl. Mech. Rev. 61, 010803 (2008).

15 M. K. Smith and S. H. Davis, "Instability of dynamic thermocapillary liquid layer. Part 1. Convective instabilities," J. Fluid Mech. 132, 119 (1983).

${ }^{16}$ M. K. Smith and S. H. Davis, "Instability of dynamic thermocapillary liquid layer. Part 2. Surface-wave instabilities," J. Fluid Mech. 132, 145 (1983).

${ }^{17}$ M. K. Smith, "Instability mechanisms in dynamic thermocapillary liquid layers," Phys. Fluids 29, 3182 (1986).

${ }^{18}$ R. J. Riley and G. P. Neitzel, "Instability of thermocapillary-buoyancy convection in shallow layers. Part 1. Characteristic of steady and oscillatory instabilities," J. Fluid Mech. 359, 143 (1998).

${ }^{19}$ D. Villers and J. K. Platten, "Coupled buoyancy and Marangoni convection in acetone: Experiments and comparison with numerical simulation,” J. Fluid Mech. 234, 487 (1992).

${ }^{20}$ J. Burguete, N. Mukolobwiez, F. Daviaud, N. Garnier, and A. Chiffaudel, "Buoyant-thermocapillary instabilities in extended liquid layers subjected to a horizontal temperature gradient," Phys. Fluids 13, 2773 (2001).

${ }^{21}$ V. M. Shevtsova, A. A. Nepomnyashchy, and J. C. Legros, "Thermocapillary-buoyancy convection in a shallow cavity heated from the side," Phys. Rev. E 67, 066308 (2003).

${ }^{22}$ F. Daviaud and J. M. Vince, "Traveling waves in a fluid layer subjected to a horizontal temperature gradient," Phys. Rev. E 48, 4432 (1993).

${ }^{23}$ C. L. Chan and C. F. Chen, "Effect of gravity on the instability of thermocapillary convection in a horizontal fluid layer," J. Fluid Mech. 647, 91 (2010).

${ }^{24}$ Y. Kamotani, S. Ostrach, and A. Pline, “Thermocapillary convection experiment in microgravity,” J. Heat Transfer 117, 611 (1995).

25 Y. Kamotani and S. Ostrach, "Oscillatory thermocapillary flows in open cylindrical containers induced by CO2 laser heating,” Int. J. Heat Mass Transfer 42, 555 (1999).

${ }^{26}$ D. Schwabe, A. Cramer, J. Schneider, S. Benz, and J. Metzger, "Experiments on the multi-roll-structure of thermocapillary flow in side-heated thin liquid layers," Adv. Space Res. 24, 1367 (1999).

${ }^{27}$ D. Schwabe and S. Benz, "Thermocapillary flow instabilities in an annulus under microgravity - Results of the experiment MAGIA,” Adv. Space Res. 29, 629 (2002).

${ }^{28}$ N. Mukolobwiez, A. Chiffaudel, and F. Daviaud, "Supercritical Eckhaus instability for surface-tension-driven hydrothermal waves," Phys. Rev. Lett. 80, 4661 (1998).

${ }^{29}$ N. Garnier, A. Chiffaudel, F. Daviaud, and A. Prigent, "Nonlinear dynamics of waves and modulated waves in 1D thermocapillary flows. I. General presentation and periodic solutions," Physica D 174, 1 (2003).

${ }^{30}$ N. Garnier, A. Chiffaudel, and F. Daviaud, "Nonlinear dynamics of waves and modulated waves in 1D thermocapillary flows. II. Convective/absolute transitions," Physica D 174, 30 (2003).

${ }^{31}$ B. M. Carpenter and G. M. Homsy, "High Marangoni number convection in a square cavity: Part II," Phys. Fluids A 2, 137 (1990).

32 J. Xu and A. Zebib, "Oscillatory two- and three-dimensional thermocapillary convection," J. Fluid Mech. 364, 187 (1998).

${ }^{33}$ C. Ma and D. Bothe, "Direct numerical simulation of thermocapillary flow based on the volume of fluid method," Int. J. Multiphase Flow 37, 1045 (2011).

${ }^{34}$ P. J. Sáenz, P. Vallui, K. Sefiane, G. Karapetsas, and O. K. Matar, "Linear and nonlinear stability of hydrothermal waves in planar liquid layers driven by thermocapillarity," Phys. Fluids 25, 094101 (2013).

35 E. Bucchignani and D. Mansutti, "Horizontal thermal convection in a shallow cavity: Oscillatory regimes and transition to chaos," Int. J. Numer. Methods Heat Fluid Flow 10, 179 (2000).

${ }^{36}$ E. Bucchignani and D. Mansutti, "Horizontal thermocapillary convection of succinonitrile: Steady state, instabilities, and transition to chaos,” Phys. Rev. E 69, 056319 (2004).

${ }^{37}$ W. R. Hu and Z. M. Tang, "Hydrothermal wave of large-Prandtl-number fluid in a shallow cavity," Sci. China, Ser. G: Phys., Mech. Astron. 50, 787 (2007).

${ }^{38}$ N. N. Vanenko, The Method of Fractional Steps (Springer-Verlag, Berlin, Heidelberg, New York, 1971).

${ }^{39}$ Z. M. Tang, K. Li, and W. R. Hu, "Influence of free surface curvature of a liquid layer on the critical Marangoni convection," Int. J. Heat Mass Transfer 51, 5102 (2008).

${ }^{40}$ P. Grassberger and I. Procaccla, "Characterization of strange attractors," Phys. Rev. Lett. 50, 346 (1983). 